\title{
Triage in the Angiography Suite for Mechanical Thrombectomy in Acute Ischemic Stroke: Not Such a Good Idea
}

T he recent evidence on the effectiveness of mechanical thrombectomy (MT) for the treatment of acute ischemic stroke (AIS) with large-vessel occlusion ${ }^{1}$ has led to rethinking of the patient workflow. Indeed, the need to rapidly bring the patients eligible for MT into the angiography suite has caused the neurologists, interventional neuroradiologists, and anesthesiologists to find new routines to minimize, as much as possible, the time between symptom onset and the recanalization by endovascular means. ${ }^{1-3}$ The main reason for this rationalization of patient management is that time to recanalization has a dramatic influence on the neurologic outcome. For instance, it has been shown in the meta-analysis of Saver et al (2016) ${ }^{1}$ that for every 4 minutes' delay in emergency department door-to-reperfusion time, 1 of every 100 treated patients had a worse disability outcome, leading to a lower benefit of MT initiated 7.3 hours after symptom onset.

One of the options proposed to reduce this critical delay is to perform the triage directly in the angiography suite. Indeed, most of the manufacturers have developed new imaging tools to obtain, in the angiography suite, via a conebeam CT acquisition with flat panel technology, unenhanced C-arm CT, C-arm CT angiography, and perfusion acquisitions using intravenous contrast material injection. Even if the accuracy of flat panel conebeam CT perfusion has still to be demonstrated, this technique may be helpful in detecting hypoperfused brain tissue that would benefit from revascularization.

This "all-inclusive" option with C-arm CT acquisitions seems, at first glance, very seductive because it reduces the delay between imaging acquisition and groin puncture and thus the revascularization time.

However, in our opinion, some limitations weaken the validity of this workflow. Indeed, in our institution (Pitié-Salpêtrière Hospital), $<30 \%$ of patients with sudden onset of neurologic deficit (ie, suspicion of acute ischemic stroke) will be eligible for mechanical thrombectomy, with the remaining patients having AIS without large-vessel occlusion or parenchymal hematoma or stroke mimics. Screening all these patients in the angiography suite would be a considerable waste of time and not cost-effective. Indeed, the average time to set up the patient on the angiography

http://dx.doi.org/10.3174/ajnr.A5610 suite table is approximately 5 minutes. The acquisition time for flat panel CT, CTA, and CT perfusion is at least 5-10 minutes. If no stroke is depicted, the angiography suite should be cleaned with a strict protocol of biocleaning (ISO7 standard) to prevent any infectious risk, because angiography suites are considered as surgical operating rooms. This biocleaning lasts at least $30 \mathrm{~min}-$ utes. Thus, for a stroke mimic or a hematoma, the angiography suite will not be available for other procedures for at least 45 minutes to 1 hour. Due to the volume of patients admitted with suspected AIS in academic centers and to the low rate of patients who finally undergo MT among them, many patients will have a "futile" imaging in the angiography suite (ie, imaging that will not lead to an endovascular procedure).

In our opinion, triage in the angiography suite could be a potentially good option only in centers with a low volume of elective cases and/or in centers where the MR imaging or CT is rarely available or very distant geographically from the angiography suite.

One could argue that a dedicated angiography suite only for MT could be an option to overcome this limitation. However, it would not be cost-effective because the flow of patients with AIS requiring $\mathrm{MT}$ is highly variable.

Others would advocate that patients should be referred directly to the angiography suite on the basis of triage using clinical scores. ${ }^{4}$ However, it has been shown that such scores are not accurate enough to depict AIS with large-vessel occlusion. ${ }^{5}$

In conclusion, rethinking the workflow of patients with AIS who need MT is a major leap forward to improve the functional outcome for these patients. However, it should not penalize the other patients treated in our hospitals. These improved workflows should remain cost-effective to be valid; our enthusiasm should not lead us to use a sledgehammer to crack a nut.

\section{ACKNOWLEDGMENTS}

The authors thank M. Pierre Grare and Bénédicte Ibert for their help in the English editing of the manuscript.

Disclosures: Frédéric Clarençon—UNRELATED: Consultancy: Balt, Medtronic, Codman Neurovascular. Nader-Antoine Sourour-UNRELATED: Consultancy: Balt, Medtronic.

AJNR Am J Neuroradiol 39:E59-E60 May 2018 www.ajnr.org 
1. Saver JL, Goyal M, van der Lugt A, et al; HERMES Collaborators. Time to treatment with endovascular thrombectomy and outcomes from ischemic stroke: a meta-analysis. JAMA 2016;316:1279-88 CrossRef Medline

2. Rinaldo L, Brinjikji W, McCutcheon BA, et al. Hospital transfer associated with increased mortality after endovascular revascularization for acute ischemic stroke. J Neurointerv Surg 2017;9:1166-72 CrossRef Medline

3. Ribo M, Boned S, Rubiera M, et al. Direct transfer to angio suite to reduce door-to-puncture time in thrombectomy for acute stroke. J Neurointerv Surg 2018;10:221-24 CrossRef Medline

4. Jadhav AP, Kenmuir CL, Aghaebrahim A, et al. Interfacility transfer directly to the neuroangiography suite in acute ischemic stroke patients undergoing thrombectomy. Stroke 2017;48:1884-89 CrossRef Medline

5. Turc G, Maier B, Naggara O, et al. Clinical scales do not reliably identify acute ischemic stroke patients with large-artery occlusion. Stroke 2016;47:1466-72 CrossRef Medline

Paris VI University, Pierre et Marie Curie Paris, France Department of Neuroradiology, Assistance Publique-Hôpitaux de Paris Pitié-Salpêtrière Hospital Paris, France (1)C. Rosso Paris VI University, Pierre et Marie Curie Paris, France Urgences Cérébro-Vasculaires, Assistance Publique-Hôpitaux de Paris Pitié-Salpêtrière Hospital Paris, France INSERM U 1127, CNRS UMR 7225 Sorbonne Universités, UPMC Univ Paris 06 UMR S 1127 Institut du Cerveau et de la Moelle Épinière, ICM, F-75013 Paris, France
Paris VI University, Pierre et Marie Curie

Paris, France

Department of Anesthesiology, Assistance Publique-Hôpitaux de Paris Pitié-Salpêtrière Hospital

Paris, France

(D) E. Shotar

Paris VI University, Pierre et Marie Curie

Paris, France

Department of Neuroradiology, Assistance Publique-Hôpitaux de Paris Pitié-Salpêtrière Hospital

Paris, France

(1) C. Rolla-Bigliani

Department of Neuroradiology, Assistance Publique-Hôpitaux de Paris Pitié-Salpêtrière Hospital

Paris, France

(D) Y. Samson

Paris VI University, Pierre et Marie Curie Paris, France

INSERM U 1127, CNRS UMR 7225

Sorbonne Universités, UPMC Univ Paris 06 UMR S 1127 Institut du Cerveau et de la Moelle Épinière, ICM, F-75013

Paris, France

Department of Anesthesiology, Assistance Publique-Hôpitaux de Paris

Pitié-Salpêtrière Hospital

Paris, France

(D). Alamowitch

Paris VI University, Pierre et Marie Curie

Paris, France

Department of Vascular Neurology Saint-Antoine Hospital

Paris, France

(1) N.-A. Sourour

Department of Neuroradiology, Assistance Publique-Hôpitaux de Paris Pitié-Salpêtrière Hospital Paris, France 